\title{
An Adaptive Second Order Fuzzy Neural Network for Nonlinear System Modeling
}

\author{
Hong-Gui Han ${ }^{\mathrm{a}, \mathrm{b}, *}$, Lu-Ming Ge ${ }^{\mathrm{a}, \mathrm{b}}$, Jun-Fei Qiao ${ }^{\mathrm{a}, \mathrm{b}}$ \\ ${ }^{a}$ College of Electronic and Control Engineering, Beijing University of Technology, Beijing, 100124, China. \\ ${ }^{\mathrm{b}}$ Beijing Key Laboratory of Computational Intelligence and Intelligent System, Beijing, 100124, China. \\ E-mail address: Rechardhan@sina.com (H. G. Han), geluming1992@126.com (L. M. Ge), and isibox@sina.com (J. F. Qiao). \\ * Corresponding author. Phone +861067391631 Fax +861067391631
}

\begin{abstract}
In this paper, an adaptive second order algorithm (ASOA) has been developed to train the fuzzy neural network (FNN) to achieve fast and robust convergence for nonlinear system modeling. Different from recent studies, this ASOA-based FNN (ASOA-FNN) has the quasi Hessian matrix and gradient vector which are accumulated as the sum of related sub matrices and vectors, respectively. Meanwhile, the learning rate of ASOA-FNN is designed to accelerate the learning speed. In addition, the convergence of the proposed ASOA-FNN has been proved both in the fixed learning rate phase and the adaptive learning rate phase. Finally, several comparisons have been realized and they have shown that the proposed ASOA-FNN has faster convergence speed and more accurate results than that of some existing methods.
\end{abstract}

Index Terms-Nonlinear system modeling; fuzzy neural network; adaptive second-order algorithm; fast convergence.

\section{INTRODUCTION}

$\mathrm{M}$ OST real processes are characterized by nonlinear and time-varying behavior, and thus there always exist modeling inaccuracies [1]-[2]. In fact, nonlinear system model imprecision may come from actual uncertainties about the plant or from the plant dynamics. However, for a nonlinear system, it is a challenge to obtain an accurate and faithful mathematical model due to its strongly nonlinear behavior, high degree of uncertainty, or time-varying characteristics [3]-[4]. Therefore, lots of attention has been paid to effective process modeling techniques for nonlinear system [5]. Recent research results show that the fuzzy neural networks (FNN), combining the capability of fuzzy reasoning to handle uncertain information and that of artificial neural networks to learn from input-output datasets, has been recognized as a powerful tool for nonlinear system modeling [6]. Based on the neural implementation of a fuzzy system, the fuzzy rules derivation and the parameters automatic tuning can be realized by using the learning and optimization methods [7].

In fact, the learning algorithm is a fundamental element in the use of FNN. Therefore, many algorithms have already been developed in recent years. Among these algorithms, the backpropagation (BP) algorithm, a powerful training technique, is probably the most frequently used technique [8]. In the BP algorithm, the descent approach is used to minimize the error 
function, and thus the local minima is always reached associated with excessively long training time for convergence [9]. To improve the convergence speed, some adaptive learning algorithms have been studied. For example, Meng et al. introduced a decreasing function to update learning rate in [10]. And a fuzzy conjugate gradient algorithm has been developed to speed up the convergence rate of FNN in [11]. However, as widely reported in the literature, these BP algorithms [9]-[11] still have limited accuracy and often suffer from local minima problem.

To improve the accuracy and avoid tracking into local minima, the evolutionary algorithms, which can find the global solution to optimize the overall parameters of FNN, have been widely used [12]-[14]. For example, Tzeng proposed an efficient genetic algorithm (GA) to adjust the parameters of FNNs. In this algorithm, the parameters such as the weights and membership functions can be characterized by minimizing a quadratic measure of the error function. The simulation results illustrate the advantages of high approximation accuracy and good generalization performance [12]. Then, a two-phase learning method, based on the typical GA, was developed to reduce the generated error of FNN in [13]. The first phase roughly estimated the optimal fuzzy weights, and the second phase provided better estimate for the shape of the membership function. Moreover, Kuo et al. designed a particle swarm optimization (PSO), according to fuzzy neural network, to determine the relationship between the radio frequency identification signals and the position of a picking cart [14]. Furthermore, some other evolutionary algorithms (such as artificial bee colony (ABC) algorithm, differential evolution (DE), Hierarchical fair competition parallel genetic algorithm, and so on) were employed to optimize the parameters of FNN in [15]-[18]. However, even though these evolutionary algorithms can find the global solution to train the parameters of FNN [12]-[18], the searching processes are extremely time-consuming [19]-[20]. In order to improve the learning speed and the solution accuracy, a species-based improved electromagnetism-like mechanism (SIEM) is proposed to optimize the parameters of FNN. This SIEM algorithm combines the advantages of electromagnetism-like mechanism and gradient-descent technique to obtain fast convergence and low computational complexity [21]. Zhao et al. proposed a new gradient learning approach for FNN, which considers both nonlinear parameters in the rule premises and linear parameters in the rule consequents. As a result, the learning speed and the accuracy of the proposed method can be improved simultaneously [22]. Moreover, a fast gradient training algorithm was used to train the parameters of FNN in [23], and a gradient learning algorithm with a dynamic learning rate was derived to adjust the parameters of the fully connected FNN in [24]. In addition, some other learning algorithms have been proposed in [25]-[27]. These gradient learning algorithms [21]-[27] lead to significantly improved modelling performance of FNN. However, most of the former algorithms have not examined the algorithm convergence that ensures the system modeling performance.

To avoid the aforementioned problems, in this paper, a hybrid learning algorithm, which is a combination of both adaptive 
second-order algorithm (ASOA) and adaptive learning rate strategy, is applied to train the parameters of FNN. This ASOA-based FNN (ASOA-FNN) has fast convergence in learning process and high efficiency to deal with nonlinear system modeling. The proposed ASOA-FNN owns four major advantages over other networks as follows: Firstly, a new ASOA is developed for training the parameters of FNN. One of the key factors of the ASOA strategy is that the quasi Hessian matrix and gradient vector are accumulated as the sum of related sub matrices and vectors, respectively. Due to the advantages of fast convergence and powerful searching ability of the proposed ASOA, this ASOA-FNN can reduce the computational complexity of the learning process and reach smaller testing error with much faster speed. Secondly, with the purpose of achieving effluent modeling performance, an adaptive learning rate strategy has been carried out for ASOA-FNN. In fact, when the iterative sequence is near the solution set, the learning rate may be smaller than the machine precision, and so it will lose its role [28]-[29]. Thus, this proposed adaptive learning rate strategy can accelerate the convergence of ASOA-FNN in the learning process. Thirdly, as the convergence is important to the applications, this proposed ASOA-FNN has been specifically designed with these in mind. The convergence analysis and convergence conditions of ASOA-FNN are demonstrated theoretically in details.

The remainder of this paper is organized as follows. Section II defines the problem and necessary conditions. The details of ASOA-FNN is introduced in Section III. Section IV discusses the convergence of ASOA-FNN and gives the convergence conditions. Section V presents the results by utilizing three types of dynamic system modeling, respectively. The models include the Mackey-Glass time-series prediction, the classical MIMO nonlinear system modeling, and the sludge bulking prediction in wastewater treatment process. Finally, Section VI concludes the paper.

\section{Problem DEFINITION}

For a multi-input and multi-output system, the nonlinear form of the system is defined by

$$
\mathbf{y}=f(\mathbf{x}),
$$

where $\mathbf{y}=\left[y_{1}, y_{2}, \ldots, y_{Q}\right]^{T}$ and $\mathbf{x}=\left[x_{1}, x_{2}, \ldots, x_{k}\right]$ are the output and input of the nonlinear system, respectively.

To model the MIMO nonlinear system, a MIMO FNN is introduced. An illustrative FNN structure is shown in Fig.1 (a MIMO for example). The mathematical description of this MIMO FNN is given below:

$$
\hat{\mathbf{g}}=\mathbf{W v}
$$

where

$$
\hat{\mathbf{g}}=\left[\hat{g}_{1}, \hat{g}_{2}, \cdots, \hat{g}_{Q}\right]^{\mathbf{T}}, \quad \mathbf{W}=\left[\mathbf{w}^{1}, \mathbf{w}^{2}, \cdots, \mathbf{w}^{Q}\right]^{\mathbf{T}}
$$


$\hat{g}_{q}$ is the output of the $q$ th neuron in the output layer, $\mathbf{W}$ is the parameter matrix, $\mathbf{w}^{q}=\left[w_{1}^{q}, w_{2}^{q}, \ldots, w_{P}^{q}\right]$ are the weights between the $q$ th neuron in the output layer and the normalized layer, $Q$ is the number of neurons in the output layer, $\mathbf{v}$ is the output of the normalized layer, and for a fuzzy model

$$
\hat{g}_{q}=\mathbf{w}^{q} \mathbf{v}=\frac{\sum_{l=1}^{P} w_{l}^{q} e^{-\sum_{i=1}^{k} \frac{\left(x_{i}-c_{i}\right)^{2}}{2 \sigma_{i l}^{2}}}}{\sum_{j=1}^{P} e^{-\sum_{i=1}^{k} \frac{\left(x_{i}-c_{i j}\right)^{2}}{2 \sigma_{i j}^{2}}}}
$$

where $P$ is the number of neurons in the normalized layer, $v_{l}$ is the output of the $l$ th normalized neuron, and $\mathbf{v}=\left[v_{1}, v_{2}, \ldots, v_{P}\right]^{\mathbf{T}}$, and

$$
v_{l}=\frac{\phi_{l}}{\sum_{j=1}^{P} \phi_{j}}=\frac{e^{-\sum_{i=1}^{k} \frac{\left(x_{i}-c_{i}\right)^{2}}{2 \sigma_{i l}^{2}}}}{\sum_{j=1}^{P} e^{-\sum_{i=1}^{k} \frac{\left(x_{i}-c_{i j}\right)^{2}}{2 \sigma_{i j}^{2}}}}, \quad j=1,2, \cdots, P ; l=1,2, \cdots, P,
$$

the number of neurons in the radial basis function (RBF) layer is equal to the number of neurons in the normalized layer, and $\phi_{j}$ is the output value of the $j$ th RBF neuron

$$
\phi_{j}=\prod_{i=1}^{k} A_{j}^{i}\left(x_{j}\right)=\prod_{i=1}^{k} e^{-\frac{\left(x_{i}-c_{i j}\right)^{2}}{2 \sigma_{i j}}}=e^{-\sum_{i=1}^{k} \frac{\left(x_{i}-c_{i j}\right)^{2}}{2 \sigma_{i j}}}, i=1,2, \cdots, k ; j=1,2, \cdots, P,
$$

$\mathbf{c}_{j}=\left[c_{1 j}, c_{2 j}, \ldots, c_{k j}\right]$ and $\boldsymbol{\sigma}_{j}=\left[\sigma_{1 j}, \sigma_{2 j}, \ldots, \sigma_{k j}\right]$ are the vectors of centers and widths of the $j$ th RBF neuron, respectively, and

$$
u_{i}=x_{i},(i=1,2, \cdots, k)
$$

where $k$ is the number of neurons in the input layer, $\mathbf{x}=\left[x_{1}, x_{2}, \ldots, x_{k}\right]$ is the input of the input layer, and $\mathbf{U}=\left[u_{1}, u_{2}, \ldots, u_{k}\right]$ is the input of the RBF layer.

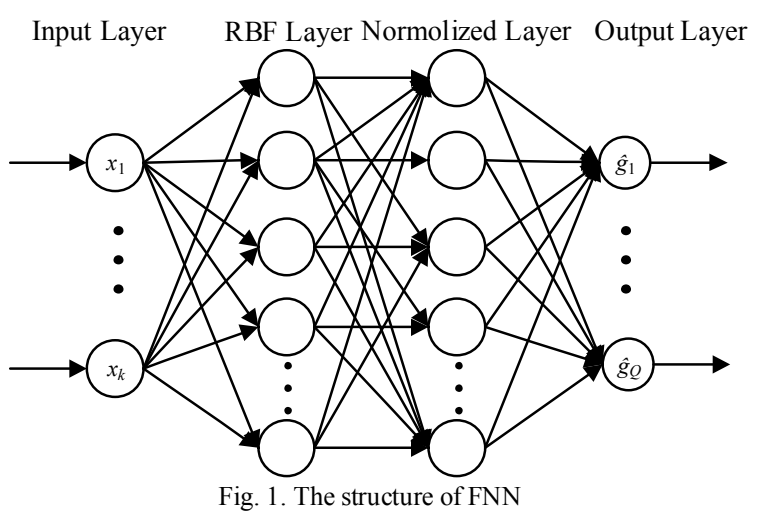

In general, the fuzzy rules of this FNN can be written as: $R_{i}$ : IF $x_{1}$ is $A_{1}^{i}, x_{2}$ is $A_{2}^{i}, \ldots, x_{k}$ is $A_{k}^{i}$; THEN $\hat{g}_{i}=\mathbf{w}^{i} \mathbf{v}$. Without loss 
of generality, the goal of this MIMO FNN is to model the nonlinear systems. It is desired to develop an adaptive learning algorithm to adjust the parameters of FNN.

\section{AdAPtive SECOND-ORder Algorithm-BASEd FuZZY-NEURAL-Network (ASOA-FNN)}

In this section, an ASOA, integrating a second-order learning algorithm and an adaptive learning rate strategy, is used to adjust the parameters of FNN (such as centers, widths, and output weights). Since the second-order learning algorithm and the adaptive learning rate strategy perform simultaneously, the proposed ASOA-FNN leads to fast convergence and high accuracy.

\section{A. Adaptive Second Order Algorithm (ASOA)}

Following the computation procedure in the Levenberg-Marquardt algorithm [30], the updated rule of ASOA is given by

$$
\boldsymbol{\Theta}(t+1)=\boldsymbol{\Theta}(t)+(\mathbf{\Psi}(t)+\lambda(t) \times \mathbf{I})^{-1} \times \mathbf{\Omega}(t),
$$

where $\boldsymbol{\Psi}(t)$ is the quasi Hessian matrix, $\mathbf{\Omega}(t)$ is gradient vector, $\mathbf{I}$ is the identity matrix which is employed to avoid the ill condition in solving inverse matrix, and $\lambda(t)$ is the adaptive learning rate defined as

$$
\begin{gathered}
\lambda(t)=\mu(t) \lambda(t-1), \\
\mu(t)=\left(\tau^{\min }(t)+\lambda(t-1)\right) /\left(\tau^{\max }(t)+1\right),
\end{gathered}
$$

where $\tau^{\max }(t)$ and $\tau^{\min }(t)$ are the maximum and minimum eigenvalues of $\Psi(t)$, respectively $\left(0<\tau^{\min }(t)<\tau^{\max }(t), 0<\lambda(t)<1,\right)$ and the variable vector $\boldsymbol{\Theta}(t)$ contains three kinds of variables: the output parameter matrix $\mathbf{W}$, the center vector $\mathbf{c}$, and the width vector

\section{$\sigma$}

$$
\boldsymbol{\Theta}(1)=\left[\mathbf{w}^{1}(1), \cdots, \mathbf{w}^{q}(1), \cdots, \mathbf{w}^{Q}(1), \mathbf{c}_{1}(1), \cdots, \mathbf{c}_{j}(1), \cdots, \mathbf{c}_{P}(1), \boldsymbol{\sigma}_{1}(1), \cdots, \boldsymbol{\sigma}_{j}(1), \cdots, \boldsymbol{\sigma}_{P}(1)\right] .
$$

In this ASOA, the output parameter matrix $\mathbf{W}$, the center vector $\mathbf{c}$, and the width vector $\boldsymbol{\sigma}$ can be optimized simultaneously. The quasi Hessian matrix $\boldsymbol{\Psi}(t)$ and the gradient vector $\boldsymbol{\Omega}(t)$ are accumulated as the sum of related sub matrices and vectors.

$$
\begin{aligned}
& \boldsymbol{\Psi}(t)=\sum_{q=1}^{Q} \boldsymbol{\Psi}_{q}(t), \\
& \mathbf{\Omega}(t)=\sum_{q=1}^{Q} \boldsymbol{\omega}_{q}(t),
\end{aligned}
$$

where the sub matrices $\boldsymbol{\psi}_{q}(t)$ and the sub vectors $\boldsymbol{\omega}_{q}(t)$ are

$$
\begin{gathered}
\boldsymbol{\psi}_{q}(t)=\mathbf{j}_{q}^{T}(t) \mathbf{j}_{q}(t), \\
\boldsymbol{\omega}_{q}(t)=\mathbf{j}_{q}^{T}(t) e_{q}(t), \\
e_{q}(t)=y_{q}(t)-\hat{g}_{q}(t),
\end{gathered}
$$

where $e_{q}(t)$ is the error of the $q$ th neuron in the output layer at time $t$, and the Jacobian vector $\mathbf{j}_{q}(t)$ is calculated as 


$$
\mathbf{j}_{q}(t)=\left[\frac{\partial e_{q}(t)}{\partial \mathbf{w}^{1}(t)}, \cdots, \frac{\partial e_{q}(t)}{\partial \mathbf{w}^{q}(t)}, \cdots, \frac{\partial e_{q}(t)}{\partial \mathbf{w}^{Q}(t)}, \frac{\partial e_{q}(t)}{\partial \mathbf{c}_{1}(t)}, \cdots, \frac{\partial e_{q}(t)}{\partial \mathbf{c}_{j}(t)}, \cdots, \frac{\partial e_{q}(t)}{\partial \mathbf{c}_{P}(t)}, \frac{\partial e_{q}(t)}{\partial \mathbf{\sigma}_{1}(t)}, \cdots, \frac{\partial e_{q}(t)}{\partial \boldsymbol{\sigma}_{j}(t)}, \cdots \frac{\partial e_{q}(t)}{\partial \boldsymbol{\sigma}_{P}(t)}\right] .
$$

Following the computation procedure in [29], the elements of the Jacobian vector $\mathbf{j}_{q}(t)$ are given as

$$
\begin{gathered}
\frac{\partial e_{q}(t)}{\partial \mathbf{w}^{q}(t)}=\left[\frac{\partial e_{q}(t)}{\partial w_{1}^{q}(t)}, \frac{\partial e_{q}(t)}{\partial w_{2}^{q}(t)}, \cdots, \frac{\partial e_{q}(t)}{\partial w_{P}^{q}(t)}\right], \\
\frac{\partial e_{q}(t)}{\partial w_{p}^{q}(t)}=-v_{p}(t), \quad p=1,2, \cdots, P, \\
\frac{\partial e_{q}(t)}{\partial \mathbf{c}_{j}(t)}=\left[\frac{\partial e_{q}(t)}{\partial c_{1 j}(t)}, \frac{\partial e_{q}(t)}{\partial c_{2 j}(t)}, \cdots, \frac{\partial e_{q}(t)}{\partial c_{k j}(t)}\right], \\
\frac{\partial e_{q}(t)}{\partial c_{i j}(t)}=-\frac{2 w_{j}^{q}(t) \times v_{i}(t) \times\left[x_{i}(t)-c_{i j}(t)\right]}{\sigma_{i j}(t)}, \quad i=1,2, \cdots, k, \\
\frac{\partial e_{q}(t)}{\partial \sigma_{j}(t)}=\left[\frac{\partial e_{q}(t)}{\partial \sigma_{1 j}(t)}, \frac{\partial e_{q}(t)}{\partial \sigma_{2 j}(t)}, \cdots, \frac{\partial e_{q}(t)}{\partial \sigma_{k j}(t)}\right], \\
\frac{\partial e_{q}(t)}{\partial \sigma_{i j}(t)}=-\frac{w_{j}^{q}(t) \times v_{i}(t) \times\left\|x_{i}(t)-c_{i j}(t)\right\|^{2}}{\sigma_{i j}^{2}(t)} .
\end{gathered}
$$

With Eqs. (18)-(23), all the elements of the Jacobian vector $\mathbf{j}_{q}(t)$ can be calculated. Then, the quasi Hessian matrix $\boldsymbol{\Psi}(t)$ and the gradient vector $\mathbf{\Omega}(t)$ are obtained from Eqs. (12)-(13), so as to apply the updated rule (8) to parameter adjustment. From the former analysis, some remarks are emphasized.

Remark 1: Second-order algorithms are in fact widely used for training the parameters of FNN [21]-[23]. However, different from the existing methods, the updated rule of the proposed ASOA is adjusted according to the Jacobian vector as shown in Eq. (17), which can be computed directly without Jacobian matrix multiplication and storage to reduce the computational complexity of the learning process. In other words, during the implementation of the proposed ASOA, matrix operations are replaced by the vector operations, which leads to significant memory reduction and speed benefit.

Remark 2: In the adaptive rules (9)-(10), a good choice of the learning rate $\lambda(t)$ helps to speed up the convergence and improve the learning performance of ASOA. It may be expensive to computer the maximum and minimum eigenvalues of Hessian matrix for adjusting the learning rate $\lambda(t)$. However, incorporating a learning rate into the learning algorithm can accelerate the convergence rate and improve the performance of ASOA-FNN. These characteristics will be illustrated in Section V.

\section{B. Adaptive Second-Order Algorithm-based Fuzzy-Neural-Network (ASOA-FNN)}

This section describes the details of ASOA-FNN which can adjust the output parameter matrix $\mathbf{w}$, the center vector $\mathbf{c}$, and 
the width vector $\boldsymbol{\sigma}$ during the training process. Root mean square error (RMSE), defined to evaluate the performance of the training process, is calculated by

$$
E(t)=\sqrt{\frac{1}{N} \sum_{t=1}^{N} \sum_{p=1}^{P}\left(e_{p}(t)\right)^{2}},
$$

where $N$ is the number of samples, and $e_{q}(t)$ is the error of the $q$ th neuron in the output layer as defined in Eq. (16).

In order to explain the training process of the proposed ASOA-FNN according to the above analysis, the main procedure is summarized in Table 1.

TABLE 1. THE TRAINING PROCESS OF ASOA-FNN

For the nonlinear system (1), create an initial FNN. Initialize the parameters $(\mathbf{w}, \mathbf{c}, \boldsymbol{\sigma})$ and the adaptive learning rate $\lambda$. The number of samples is $N$.

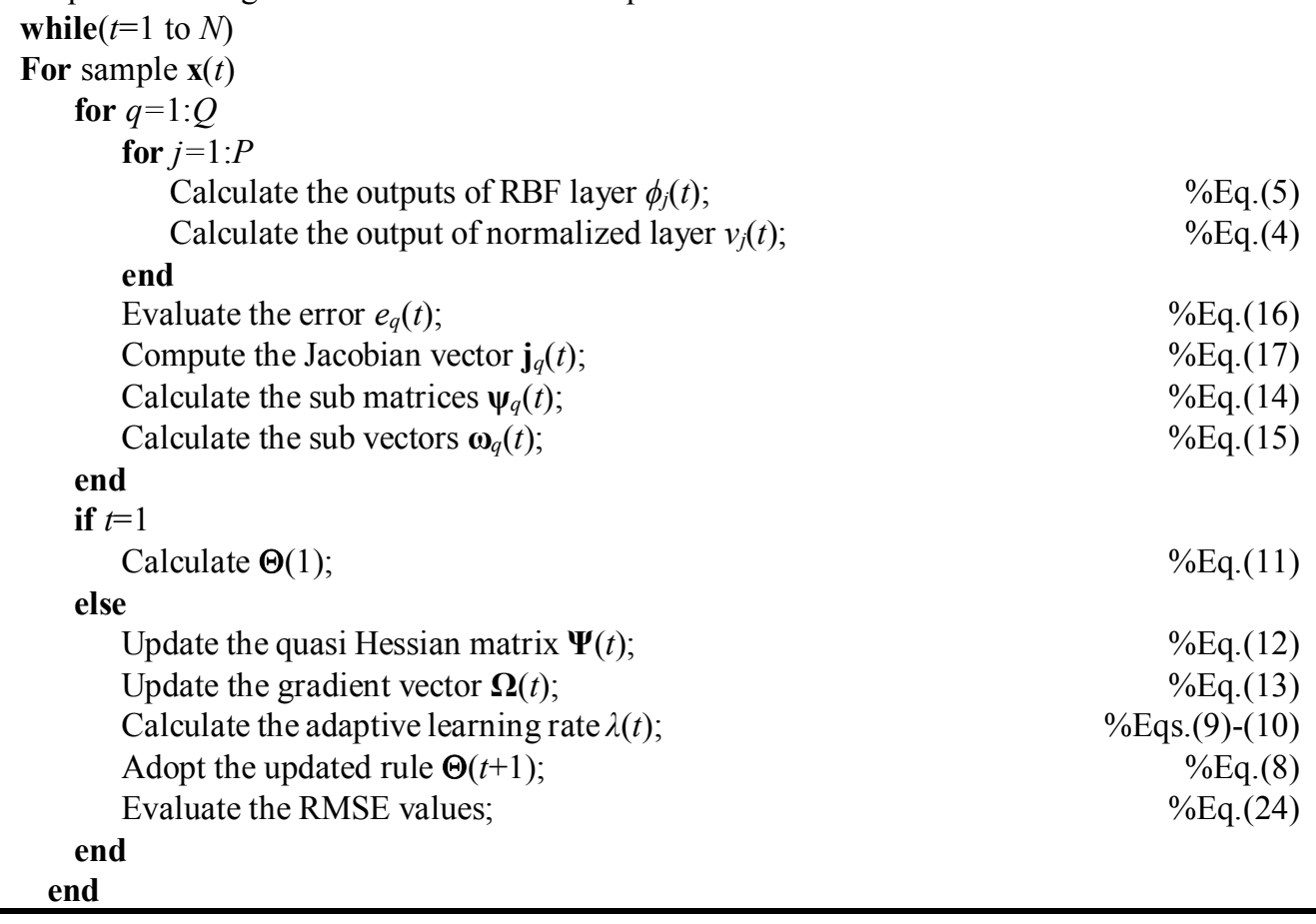

Remark 3: With the proposed training algorithm, this ASOA-FNN can improve the performances, such as the training speed and the modeling accuracy. The ASOA-based training process can be treated as the basis for an integrated FNN training methodology.

Remark 4: It can be seen that the proposed ASOA-FNN is an online algorithm. Since the network is continuously re-trained, the training time is very important to ASOA-FNN. The key issue of the proposed ASOA-FNN is to adopt the updated rule $\Theta$ in the training process. For the learning process requires only the Jacobian vector $\mathbf{j}_{q}$ and the adaptive learning rate $\lambda$, the proposed method may also shorten the computation time in many cases. 


\section{Convergence Proof And Discussion}

For the proposed ASOA-FNN, the convergence of the learning process is an important index and it requires careful investigation for successful applications. In this section, the convergence of ASOA-FNN is firstly determined with a fixed learning rate. Then, since the proposed ASOA-FNN is affected by the learning rate, the convergence of ASOA-FNN in the case with the adaptive learning rate is discussed. Through these theoretical analyses, one can get better understanding of ASOA-FNN.

\section{A. Convergence Analysis for ASOA-FNN}

For convenient discussion, the modeling error of ASOA-FNN is introduced as:

$$
\mathbf{e}(t)=\hat{\mathbf{g}}(t)-\mathbf{y}(t)
$$

where $\mathbf{e}(t)=\left[e_{1}(t), e_{2}(t), \ldots, e_{P}(t)\right]^{\mathbf{T}}$, and $\hat{\mathbf{g}}(t)$ is the output of ASOA-FNN and $\mathbf{y}(t)$ is the output of the nonlinear systems at time $t$.

Theorem 1 (Convergence of ASOA-FNN with a fixed learning rate). ASOA-FNN is trained by the updated rule defined by Eq. (8), if

$$
\begin{gathered}
\|\Delta \boldsymbol{\Theta}(t)\| \leq \kappa(t), \\
\kappa(t)=\min \left\{\|\Delta \boldsymbol{\Theta}(t-1)\|, \frac{\|\boldsymbol{\Omega}(\boldsymbol{\Theta}(t-1))\|}{\|\boldsymbol{\Psi}(\boldsymbol{\Theta}(t-1))\|}\right\},
\end{gathered}
$$

the convergence of ASOA-FNN can be guaranteed as follows

$$
\lim _{t \rightarrow \infty} \mathbf{e}(t)=\mathbf{0}
$$

Proof. Now define the Lyapunov function candidate as:

$$
V(\boldsymbol{\Theta}(t))=\frac{1}{2} \mathbf{e}^{T}(t) \mathbf{e}(t),
$$

the change of the Lyapunov function $V(\Theta(t))$ is obtained according to Refs. [30]-[31]

$$
\Delta V(\boldsymbol{\Theta}(t))=V(\boldsymbol{\Theta}(t+1))-V(\boldsymbol{\Theta}(t))=-\nabla \mathbf{E}^{T}(\boldsymbol{\Theta}(t)) \Delta \boldsymbol{\Theta}(t)+\frac{1}{2} \Delta \boldsymbol{\Theta}^{T}(t) \nabla^{2} \mathbf{E}(\boldsymbol{\Theta}(t)) \Delta \boldsymbol{\Theta}(t),
$$

where $\nabla \mathbf{E}(\Delta \boldsymbol{\Theta}(t))$ and $\nabla^{2} \mathbf{E}(\Delta \boldsymbol{\Theta}(t))$ are the matrix of first-order and second-order derivatives of the cost function, respectively. Using the updated rule as defined in Eq. (8), there will be

$$
\begin{gathered}
\Delta \boldsymbol{\Theta}(t)=(\boldsymbol{\Psi}(\boldsymbol{\Theta}(t))+\lambda(t) \mathbf{I})^{-1} \boldsymbol{\Omega}(\boldsymbol{\Theta}(t)), \\
\nabla \mathbf{E}(\boldsymbol{\Theta}(t))=\mathbf{\Omega}(\boldsymbol{\Theta}(t)), \\
\nabla^{2} \mathbf{E}(\boldsymbol{\Theta}(t))=\boldsymbol{\Psi}(\boldsymbol{\Theta}(t))+\lambda(t) \mathbf{I} .
\end{gathered}
$$

Combining Eqs. (30) and (31)-(33), there holds the results: 


$$
\Delta V(\boldsymbol{\Theta}(t))=-\frac{1}{2} \Delta \Theta^{T}(t) \nabla^{2} \mathbf{E}(\boldsymbol{\Theta}(t)) \Delta \Theta(t)
$$

From Eq. (29), one has

$$
V(\Theta(t))>0 .
$$

When the situations (26)-(27) are satisfied, the matrix $\nabla^{2} \mathbf{E}(\Delta \Theta(t))$ is positive definite, it can be concluded that

$$
\Delta V(\Theta(t))<0 .
$$

Hence, according to the Lyapunov like lemma, the convergence of ASOA-FNN can be guaranteed and $\mathbf{e}(t) \rightarrow \mathbf{0}$ as $t \rightarrow \infty$.

By now it is proved for the convergence result of ASOA-FNN with a fixed learning rate.

Remark 5: From the former discussion, one can know that the convergence of the proposed ASOA-FNN can be maintained with a fixed learning rate in theory.

\section{B. Adaptive Learning Rate Analysis for ASOA-FNN}

The convergence of the proposed ASOA-FNN with the adaptive learning rate will be discussed in details.

Theorem 2 (Convergence of ASOA-FNN with the adaptive learning rate). Suppose that Theorem 1 is valid and let the learning rate $\lambda(t)$ of the weight optimizing strategy be defined by Eqs. (9)-(10). The ASOA-FNN learning algorithm can accelerate the convergence.

Proof. The error with different weight matrixes can be rewritten as

$$
\begin{aligned}
& V(\boldsymbol{\Theta}(t+1))=V(\boldsymbol{\Theta}(t))-\nabla \mathbf{E}^{T}(\boldsymbol{\Theta}(t)) \Delta \Theta(t)+\frac{1}{2} \Delta \boldsymbol{\Theta}^{T}(t) \nabla^{2} \mathbf{E}(\boldsymbol{\Theta}(t)) \Delta \Theta(t), \\
& \bar{V}(\boldsymbol{\Theta}(t+1))=V(\boldsymbol{\Theta}(t))-\nabla \overline{\mathbf{E}}^{T}(\boldsymbol{\Theta}(t)) \Delta \bar{\Theta}(t)+\frac{1}{2} \Delta \bar{\Theta}^{T}(t) \nabla^{2} \overline{\mathbf{E}}(\boldsymbol{\Theta}(t)) \Delta \bar{\Theta}(t),
\end{aligned}
$$

where $V(\Theta(t+1))$ and $\bar{V}(\Theta(t+1))$ are the Lyapunov function candidates of ASOA-FNN with fixed learning rate and adaptive learning rate at time $t+1$, respectively, and

$$
\begin{gathered}
\Delta \overline{\boldsymbol{\Theta}}(t)=(\boldsymbol{\Psi}(\boldsymbol{\Theta}(t))+\lambda(t-1) \mathbf{I})^{-1} \boldsymbol{\Omega}(\boldsymbol{\Theta}(t)), \\
\nabla^{2} \overline{\mathbf{E}}(\boldsymbol{\Theta}(t))=\boldsymbol{\Psi}(\boldsymbol{\Theta}(t))+\lambda(t-1) \mathbf{I},
\end{gathered}
$$

where $\Delta \Theta(t)$ and $\nabla^{2} \mathbf{E}(\Delta \Theta(t))$ are shown in equations (31) and (33). Then, from the results in Theorem 1, it is obtained

$$
\begin{aligned}
V(\boldsymbol{\Theta}(t+1))-\bar{V}(\boldsymbol{\Theta}(t+1)) & =\frac{1}{2} \Delta \bar{\Theta}^{T}(t) \nabla^{2} \mathbf{E}(\boldsymbol{\Theta}(t)) \Delta \overline{\boldsymbol{\Theta}}(t)-\frac{1}{2} \Delta \Theta^{T}(t) \nabla^{2} \mathbf{E}(\boldsymbol{\Theta}(t)) \Delta \Theta(t) \\
& =\frac{1}{2} \mathbf{\Omega}^{T}(\boldsymbol{\Theta}(t))\left[\frac{1}{\mathbf{\Psi}(\boldsymbol{\Theta}(t))+\lambda(t-1) \mathbf{I}}-\frac{1}{\boldsymbol{\Psi}(\boldsymbol{\Theta}(t))+\mu(t) \lambda(t-1) \mathbf{I}}\right] \mathbf{\Omega}(\boldsymbol{\Theta}(t)) \\
& =\frac{1}{2} \boldsymbol{\Omega}^{T}(\boldsymbol{\Theta}(t)) \frac{(\mu(t) \lambda(t-1)-\lambda(t-1))}{\nabla^{2} \overline{\mathbf{E}}(\boldsymbol{\Theta}(t)) \nabla^{2} \mathbf{E}(\boldsymbol{\Theta}(t))} \mathbf{\Omega}(\boldsymbol{\Theta}(t)) \\
& =\frac{1}{2} \lambda(t-1)(\mu(t)-1) \mathbf{\Omega}^{T}(\boldsymbol{\Theta}(t))\left[\nabla^{2} \overline{\mathbf{E}}(\boldsymbol{\Theta}(t)) \nabla^{2} \mathbf{E}(\boldsymbol{\Theta}(t))\right]^{-1} \mathbf{\Omega}(\boldsymbol{\Theta}(t)) .
\end{aligned}
$$


According to the learning rate $\lambda(t)$ of the weight optimizing strategy defined by Eqs. (9)-(10), and $0<\lambda(t)<1$, thus there exits:

$$
1-\frac{\tau^{\min }(t-1)\left(1+\tau^{\max }(t-1)\right)}{\tau^{\max }(t-1)}<\lambda(t-1) .
$$

Then, one has:

$$
\mu(t)<1
$$

Hence, by taking Eqs. (41) and (43) into consideration, it leads to

$$
V(\Theta(t+1))-\bar{V}(\Theta(t+1))<0 .
$$

The proof of Theorem 2 is completed.

By now the convergence of the proposed ASOA-FNN with the adaptive learning rate has been proved.

Remark 6: Theorems 1 and 2 show the convergence of the proposed ASOA-FNN with fixed learning rate and adaptive learning rate, respectively. Moreover, one of the key advantages of the approach is that the modeling error can uniformly asymptotically converge to zero, which means that this proposed ASOA-FNN with adaptive learning rate may improve the chance to approximate the global optimization parameters.

\section{V.ILLUSTRATIVE RESULTS}

The aim of this section is to evaluate the proposed ASOA-FNN for nonlinear system modeling. In the following experiments, the performance of ASOA-FNN is tested on three examples: the Mackey-Glass time-series prediction, the classical MIMO nonlinear system modeling, and the sludge bulking prediction in wastewater treatment process. The results are compared with some other existing methods. All the simulations were programmed with Matlab version 2010, and were run on a PC with a clock speed 2.6 GHz and 4 GB RAM, under a Microsoft Windows 8.0 environment.

The performance of the algorithms is measured by using the average percentage error (APE), which is defined as

$$
\operatorname{APE}(t)=\frac{1}{N} \sum_{t=1}^{N} \frac{\|\mathbf{e}(t)\|}{\|\mathbf{y}(t)\|} \times 100 \%
$$

and the sum of squared error (SSE)

$$
\operatorname{SSE}(t)=\sum_{t=1}^{N} \mathbf{e}^{T}(t) \mathbf{e}(t) \times 100 \%,
$$

as well as the RMSE. Moreover, there are 6 normalized neurons of ASOA-FNN and $\lambda(0)=0.01$ for all the simulations.

\section{A. Mackey-Glass Time-series Prediction}

In this example, the effectiveness of ASOA-FNN is examined by the Mackey-Glass time-series prediction, which is described as 


$$
x(t+1)=(1-a) x(t)+\frac{b x(t-\tau)}{1+x^{10}(t-\tau)},
$$

where $a=0.1, b=0.2, \tau=17$ and the initial condition $x(0)=1.2$. From $[9,18,23,25,26,27,33,34]$, the prediction model is given by

$$
x(t+P)=\bar{f}(x(t), x(t-6), x(t-12), x(t-18)) .
$$

500 data points between $t=1$ and 500 are chosen as the training samples, and another 500 data points between $t=501$ and 1000 are given as the testing samples.

The number of normalized neurons is 6 . The results are shown in Figs. 2-4. The RMSE values with the adaptive learning rate and fixed learning rate $(\lambda=0.01)$ in the training process are shown in Fig. 2. It is easily seen that the proposed ASOA-FNN with an adaptive learning rate gives a better approximation of the underlying model than that with fixed learning rate $(\lambda=0.01)$. The prediction capability of the learned ASOA-FNN are illustrated using another 500 data points between $t=501$ and 1000 . The testing results in Figs. 3-4 show that the proposed ASOA-FNN can efficiently predict the Mackey-Glass time-series no matter whether the learning rate is fixed or not.

In order to evaluate the performance of ASOA-FNN, the simulation results by using ASOA-FNN were compared with that of five other FNNs: the self-organizing fuzzy-neural-network with adaptive computation algorithm (SOFNN-ACA) [9], the correlated fuzzy neural network with Levenberg-Marquardt (CFNNLM) [25], the locally recurrent fuzzy neural network with support vector regression (LRFNN-SVR) [26], the fast and accurate online self-organizing scheme for parsimonious fuzzy neural networks (FAOS-PFNN) [34], and the growing-and-pruning fuzzy neural network (GP-FNN) [27] algorithms. Table 2 shows the comparison in terms of the testing RMSE, the testing APE, and the training time. Moreover, to clearly demonstrate the performance of ASOA-FNN with the adaptive learning rate strategy, a statistical preference analysis of ASOA-FNN with different fixed learning rates and different number of normalized neurons is also given in Table 2.

It can be seen that the proposed ASOA-FNN with adaptive learning rate (with 6 normalized neurons) gains the best testing RMSE (0.0091), the best testing APE (0.0054), and the best training time (5.29s). Then, the ASOA-FNN with adaptive learning rate (with 6 normalized neurons) achieves the best approximation and generalization performance compared with that of other algorithms. The simulation results show that the ASOA-FNN with adaptive learning rate (with 6 normalized neurons) can predict this Mackey-Glass time-series system with best approximation and generalization performance, which means an adaptive learning rate strategy is more likely to approximate the global optimization parameters of FNNs than those algorithms with the fixed learning rates. 


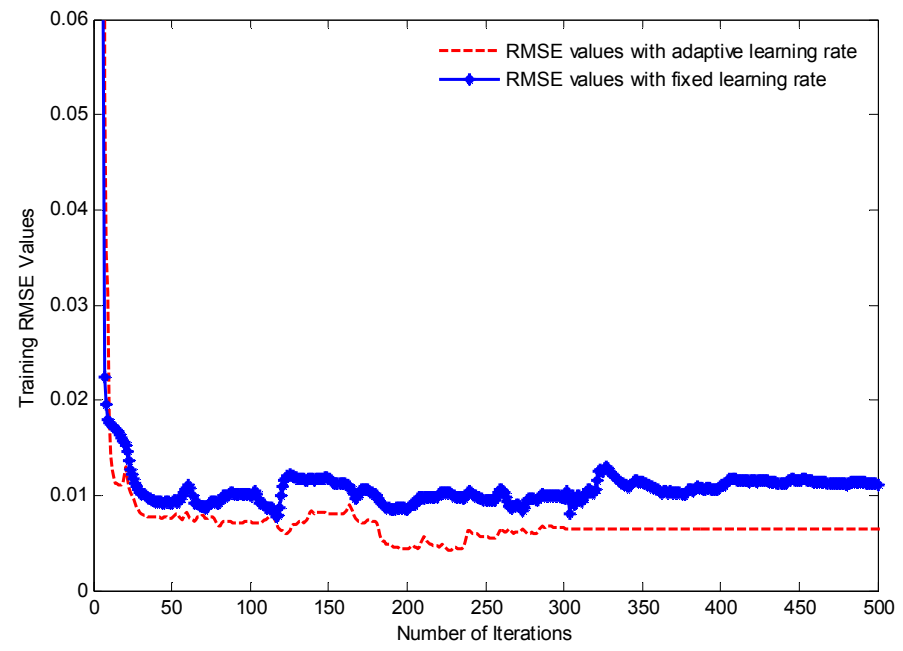

Fig. 2. RMSE values during the training process

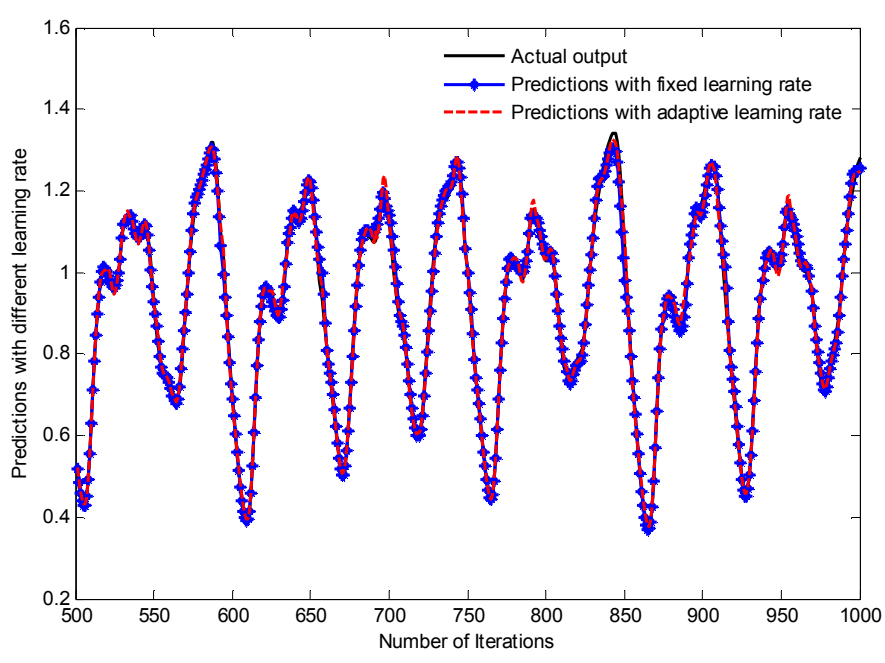

Fig. 3. Desired and predicted outputs during the testing process

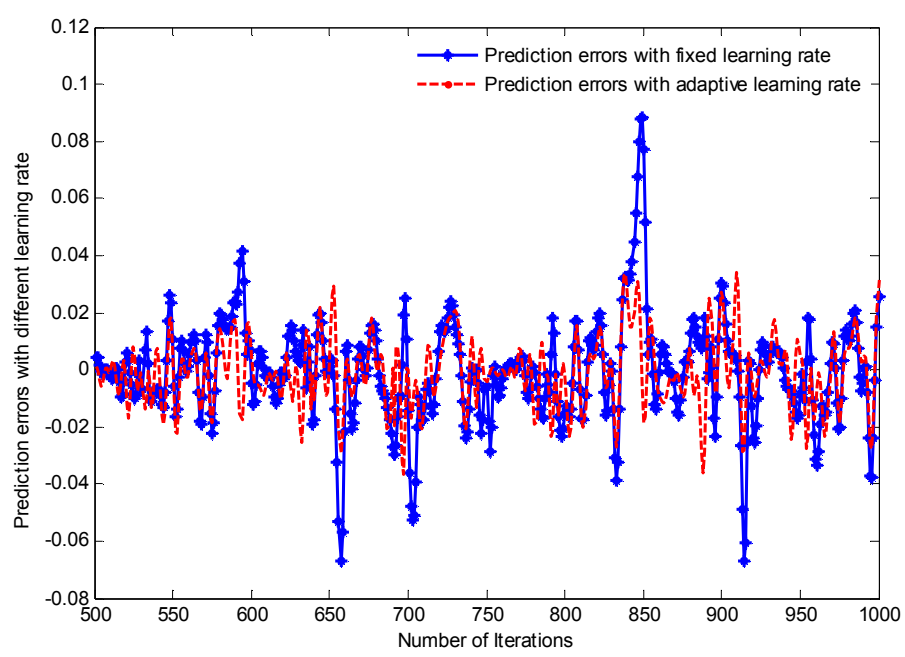

Fig. 4. Prediction error during testing process 
TABLE 2. THE PERFORMANCE COMPARISON OF DIFFERENT ALGORITHMS (ALL RESULTS WERE AVERAGED ON 30 INDEPENDENT RUNS)

\begin{tabular}{ccccc}
\hline Algorithms & Rules & Testing RMSE & Testing APE & Training time $(s)$ \\
\hline ASOA-FNN (Adaptive learning rate $\lambda(\mathbf{0})=\mathbf{0 . 0 1})$ & $\mathbf{6}$ & $\mathbf{0 . 0 0 9 1}$ & $\mathbf{0 . 0 0 5 4}$ & $\mathbf{5 . 2 9}$ \\
\hline ASOA-FNN (Adaptive learning rate $\lambda(0)=0.01)$ & 4 & 0.0102 & 0.0061 & 4.08 \\
ASOA-FNN (Adaptive learning rate $\lambda(0)=0.01)$ & 5 & 0.0103 & 0.0065 & 4.98 \\
ASOA-FNN (Adaptive learning rate $\lambda(0)=0.01)$ & 7 & 0.0095 & 0.0059 & 7.36 \\
ASOA-FNN (Adaptive learning rate $\lambda(0)=0.01)$ & 8 & 0.0095 & 0.0059 & 8.66 \\
\hline ASOA-FNN (Fixed learning rate $\lambda=0.01)$ & 6 & 0.0112 & 0.0080 & 8.56 \\
ASOA-FNN (Fixed learning rate $\lambda=0.05)$ & 6 & 0.0118 & 0.0082 & 7.89 \\
ASOA-FNN (Fixed learning rate $\lambda=0.001)$ & 6 & 0.0108 & 0.0077 & 9.12 \\
ASOA-FNN (Fixed learning rate $\lambda=0.0005)$ & 6 & 0.0110 & 0.0078 & 9.81 \\
\hline SOFNN-ACA [9] & $7 *$ & $0.0107^{*}$ & $0.0076^{*}$ & $27.33^{*}$ \\
CFNNLM [25] & $3 *$ & 0.0116 & 0.0081 & 13.32 \\
LRFNN-SVR [26] & $3^{*}$ & $0.0550^{*}$ & $0.0391^{*}$ & $72.10^{*}$ \\
FAOS-PFNN [34] & $11^{*}$ & $0.0127^{*}$ & $0.0090^{*}$ & $18.18^{*}$ \\
GP-FNN [27] & $7^{*}$ & $0.0107^{*}$ & $0.0076^{*}$ & $27.33^{*}$ \\
\hline
\end{tabular}

* The results are listed in the original papers.

\section{B. Multiple-Input Multiple-Output Nonlinear System Modeling}

To test the proposed ASOA-FNN for modeling MIMO nonlinear system, a MIMO nonlinear system is given by Eqs. (49)-(50) which has been used in several papers, notably $[32,35]$ to demonstrate the effectiveness of FNN.

$$
\begin{gathered}
y_{1}(t)=0.4 y_{1}(t-1)-0.1 y_{2}(t-1)^{3}+g_{1}(t), \\
y_{2}(t)=0.5 y_{1}(t-1)+u(t-1)+g_{2}(t),
\end{gathered}
$$

where the system inputs are $u(t)=\sin (\pi t / 25)$, and two Gaussian noise are given by $g_{1}(t) \sim N\left(0,0.01^{2}\right)$ and $g_{2}(t) \sim N\left(0,0.02^{2}\right)$, respectively. The first 500 samples from $t=1$ to $t=500$ are selected as the training sets, and another 500 samples from $t=501$ to $t=1000$ are chosen as the testing sets. The prediction values are $\mathbf{y}(t)=\left[y_{1}(t), y_{2}(t)\right]$ and the input variables are defined as

$$
\mathbf{x}(t)=\left[y_{1}(t-1), y_{1}(t-2), y_{1}(t-3), y_{2}(t-1), y_{2}(t-2), y_{2}(t-3), u(t-1), u(t-2), u(t-3)\right]
$$

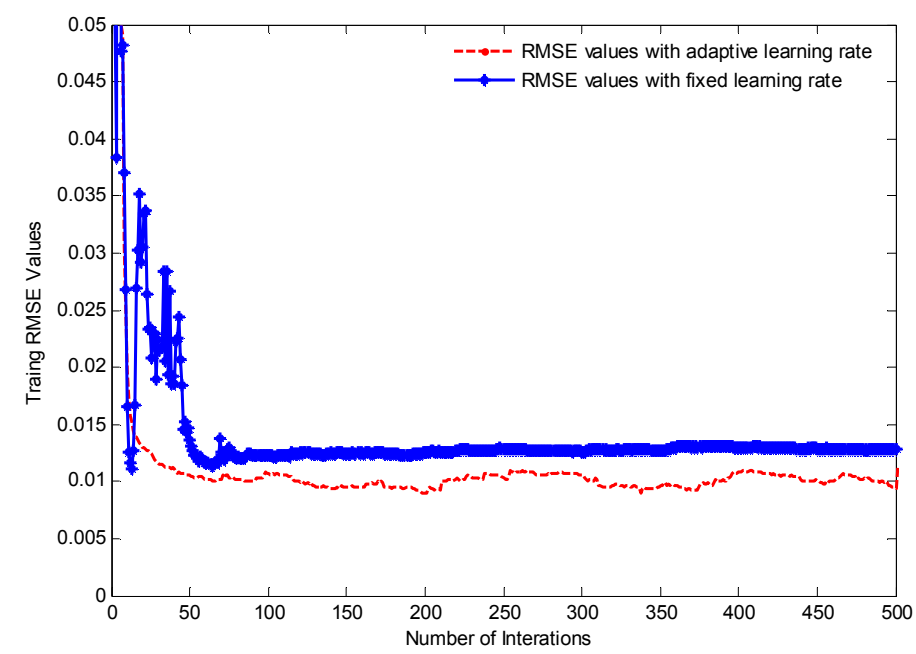

Fig. 5. RMSE values during the training process 


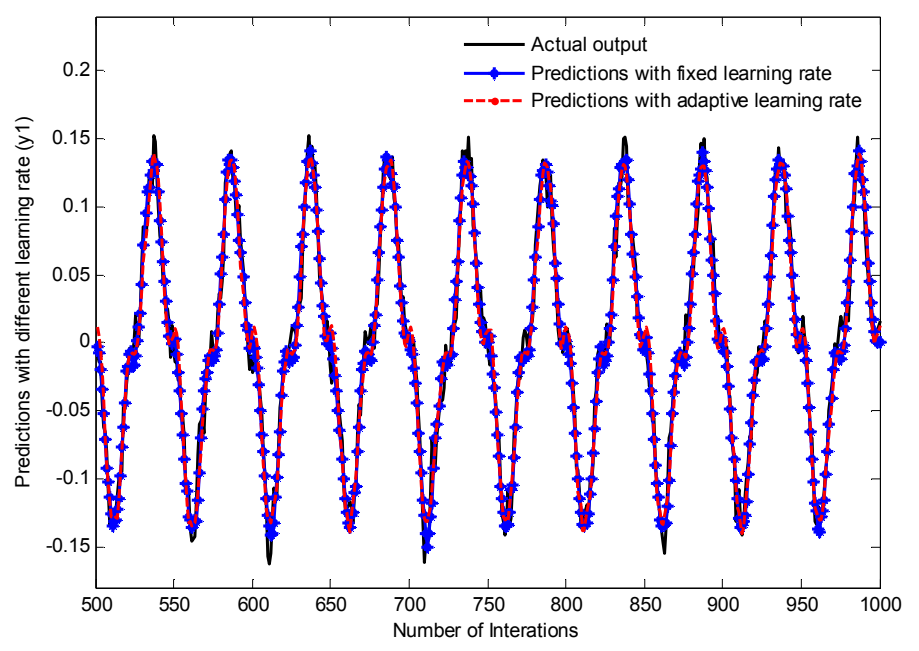

Fig. 6. Predictions of $y_{1}$ with different learning rates

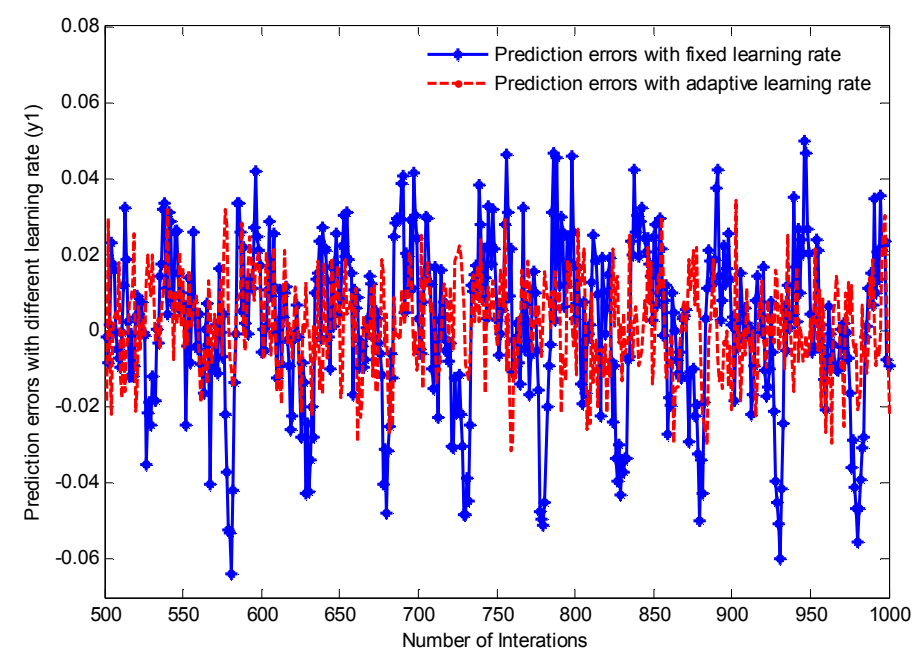

Fig. 7. Prediction errors of $y_{1}$ with different learning rates

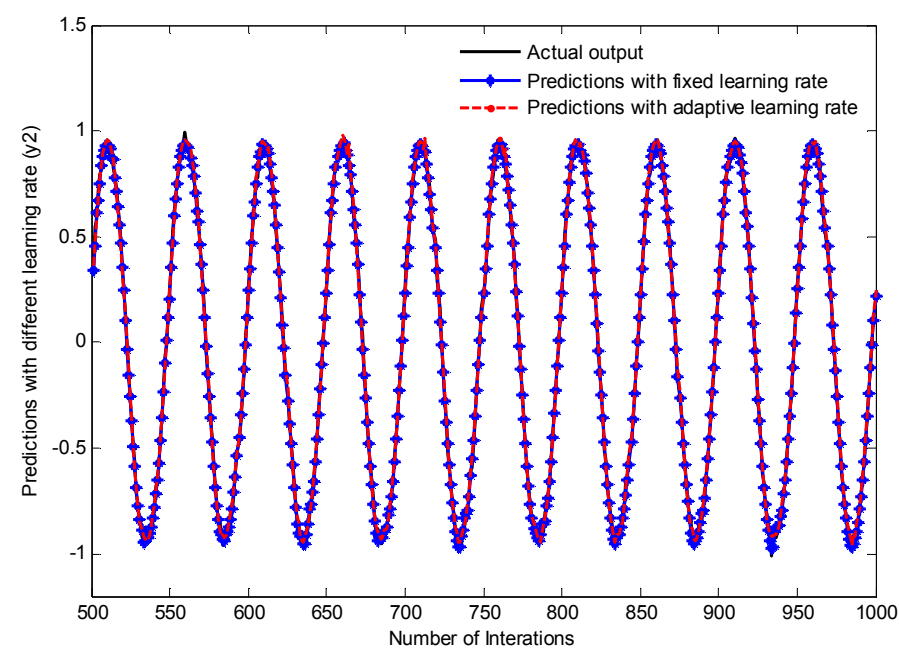

Fig. 8. Predictions of $y_{2}$ with different learning rates 


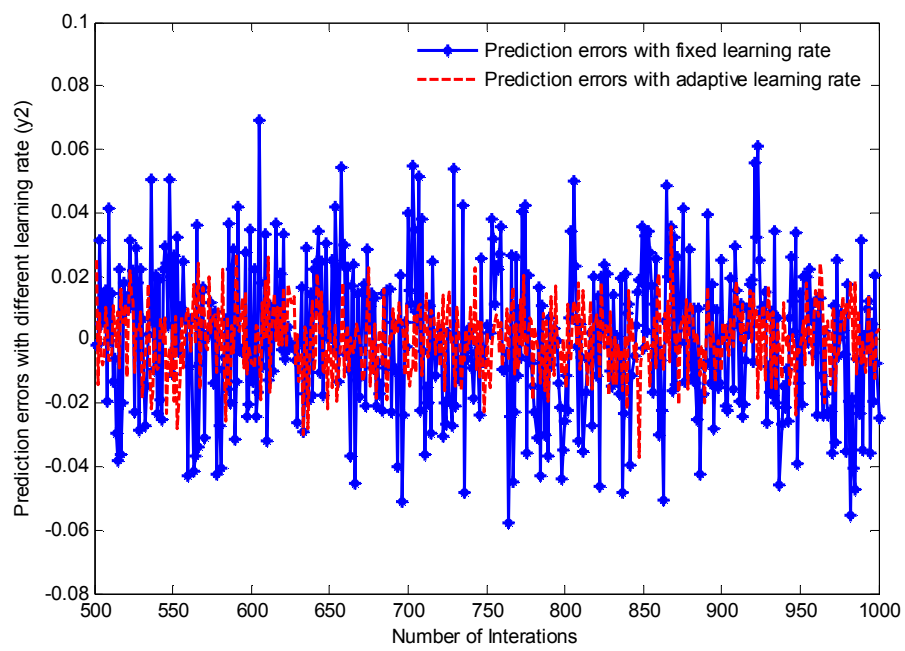

Fig. 9. Prediction errors of $y_{2}$ with different learning rates

TABLE 3. COMPARISON OF DIFFERENT ALGORITHMS FOR THE MIMO NONLINEAR SYSTEM MODELING (ALL RESULTS WERE AVERAGED ON 30 INDEPENDENT RUNS)

\begin{tabular}{|c|c|c|c|c|}
\hline Output variables & Algorithms & Rules & Testing SSE & Training time \\
\hline \multirow{13}{*}{$y_{1}$} & ASOA-FNN (Adaptive learning rate $\lambda(0)=0.01)$ & 6 & 0.045 & 4.22 \\
\hline & ASOA-FNN (Adaptive learning rate $\lambda(0)=0.01$ ) & 4 & 0.054 & 2.37 \\
\hline & ASOA-FNN (Adaptive learning rate $\lambda(0)=0.01$ ) & 5 & 0.055 & 3.32 \\
\hline & ASOA-FNN (Adaptive learning rate $\lambda(0)=0.01$ ) & 7 & 0.056 & 5.81 \\
\hline & ASOA-FNN (Adaptive learning rate $\lambda(0)=0.01$ ) & 8 & 0.063 & 6.92 \\
\hline & ASOA-FNN (Fixed learning rate $\lambda=0.01$ ) & 6 & 0.069 & 4.31 \\
\hline & ASOA-FNN (Fixed learning rate $\lambda=0.05$ ) & 6 & 0.074 & 4.29 \\
\hline & ASOA-FNN (Fixed learning rate $\lambda=0.001$ ) & 6 & 0.061 & 4.88 \\
\hline & ASOA-FNN (Fixed learning rate $\lambda=0.0005$ ) & 6 & 0.065 & 4.97 \\
\hline & SOFNN-ACA [9] & 7 & 0.042 & 37.76 \\
\hline & CFNNLM [25] & 3 & 0.086 & 0.1750 \\
\hline & TSFS-SVR [35] & $28 *$ & $0.066^{*}$ & 1.89 \\
\hline & ISO-FNN [32] & $15^{*}$ & $0.052 *$ & 5.1 \\
\hline \multirow{13}{*}{$y_{2}$} & ASOA-FNN (Adaptive learning rate $\lambda(0)=0.01$ ) & 6 & 0.12 & 4.22 \\
\hline & ASOA-FNN (Adaptive learning rate $\lambda(0)=0.01$ ) & 4 & 0.14 & 2.37 \\
\hline & ASOA-FNN (Adaptive learning rate $\lambda(0)=0.01$ ) & 5 & 0.13 & 3.32 \\
\hline & ASOA-FNN (Adaptive learning rate $\lambda(0)=0.01$ ) & 7 & 0.14 & 5.81 \\
\hline & ASOA-FNN (Adaptive learning rate $\lambda(0)=0.01$ ) & 8 & 0.15 & 6.92 \\
\hline & ASOA-FNN (Fixed learning rate $\lambda=0.01$ ) & 6 & 0.14 & 4.31 \\
\hline & ASOA-FNN (Fixed learning rate $\lambda=0.05$ ) & 6 & 0.14 & 4.29 \\
\hline & ASOA-FNN (Fixed learning rate $\lambda=0.001$ ) & 6 & 0.15 & 4.88 \\
\hline & ASOA-FNN (Fixed learning rate $\lambda=0.0005$ ) & 6 & 0.15 & 4.97 \\
\hline & SOFNN-ACA [9] & 7 & 0.12 & 37.76 \\
\hline & CFNNLM [25] & 3 & 0.17 & 28.78 \\
\hline & TSFS-SVR [35] & $28 *$ & $0.27 *$ & 1.89 \\
\hline & ISO-FNN [32] & $19 *$ & $0.22 *$ & 5.11 \\
\hline
\end{tabular}

* The results are listed in the original papers. 
In this experiment, the number of normalized neurons is also 6. The modeling results for this MIMO nonlinear system are displayed in Figs. 5-9, including the RMSE values in the training process and the predictions of the two output variables via the proposed ASOA-FNN algorithm. Fig. 5 shows that the ASOA-FNN with an adaptive learning rate is better than that with fixed learning rate $(\lambda=0.01)$. Figs. 6-7 and Figs. 8-9 illustrate the prediction values and prediction errors of $y_{1}$ and $y_{2}$, respectively. Figs. 6-9 demonstrate that the proposed ASOA-FNN algorithm can model this MIMO nonlinear system with good accuracy and generalization capability. In addition, the results are compared with that of the SOFNN-ACA [9], the CFNNLM [25], the improved structure optimization of fuzzy neural network (ISO-FNN) [32], and the Takagi-Sugeno fuzzy system-based support vector regression (TSFS-SVR) [35] algorithm. Meanwhile, the details of the comparisons of this example are given in Table 3. And, the preference of ASOA-FNN with different fixed learning rates and different number of normalized neurons is also given in Table 3 .

From Table 3, it can be seen that the proposed ASOA-FNN algorithm with adaptive learning rate (with 6 normalized neurons) needs lest training time (except the TSFS-SVR algorithm). Also, the proposed ASOA-FNN algorithm with adaptive learning rate (with 6 normalized neurons) obtains better testing RMSE ( 0.045 for $y_{1}$, and 0.12 for $\left.y_{2}\right)$. Hence, the proposed ASOA-FNN algorithm is more suitable to this MIMO nonlinear system modeling than the other methods.

\section{Sludge Bulking Prediction}

The performance of an activated sludge system for wastewater treatment processes is often deteriorated due to sludge separation problems caused by sludge bulking [36]-[37]. However, in wastewater treatment processes, it is difficult to estimate the important process variable - sludge volume index (SVI) in time due to the limitation of process technology or measurement techniques [38].

To predict SVI values accurately, a soft-computing method, based on the proposed ASOA-FNN algorithm, is developed. A number of steps are executed during the predicting development process. Fig. 10 illustrates the main steps of the overall methodology. In the experiment, the most important water quality parameters were selected as the input variables in the study. Sludge bulking is probably related to the parameters such as influent flow ( $\left.\mathrm{Q}_{\text {in }}\right)$, biological oxygen demand (BOD), chemical oxygen demand (COD), dissolved oxygen (DO) concentration, $\mathrm{pH}$, and total nutrients (TN) [38]. The inputs, the main apparatus, and instruments used in the wastewater treatment processes are listed in Table 4.

In this example, all variables are normalized and de-normalized between 0 and 1 before and after the application in the soft-computing method. This process is carried out by determining the maximum and minimum values of each variable over the whole data period. The input-output water quality data from a real wastewater treatment plant (Beijing, China) were 
measured over the year 2013. After abnormal data were deleted, 640 samples were obtained and normalized. Among them, 49 samples in October were used as test data and the remaining 591 samples were employed as training data.

For comparison, the SVI values by utilizing the soft-computing method are depicted together with the real process in Fig. 11. In order to check the predicting abilities of different methods, the errors using different methods are shown in Fig. 12. Clearly, the predicting values obtained from using the proposed ASOA-FNN model with adaptive learning rate is more accurate than that by employing the proposed ASOA-FNN model with fixed learning rate $(\lambda=0.01)$. The predicting values from ASOA-FNN are compared with those from the FNN model [7], from the ISO-FNN algorithm [32] and from the self-organizing radial basis function neural network (SORBFNN) method [38]. For fair comparison, the optimal parameters of the models are the same as that in the initial papers. Performance is assessed by using the testing RMSE and the predicting accuracy as defined in [38]. The details are presented in Table 5.



Fig. 10. Overall methodology of the ASOA-FNN-based soft-computing method for SVI 


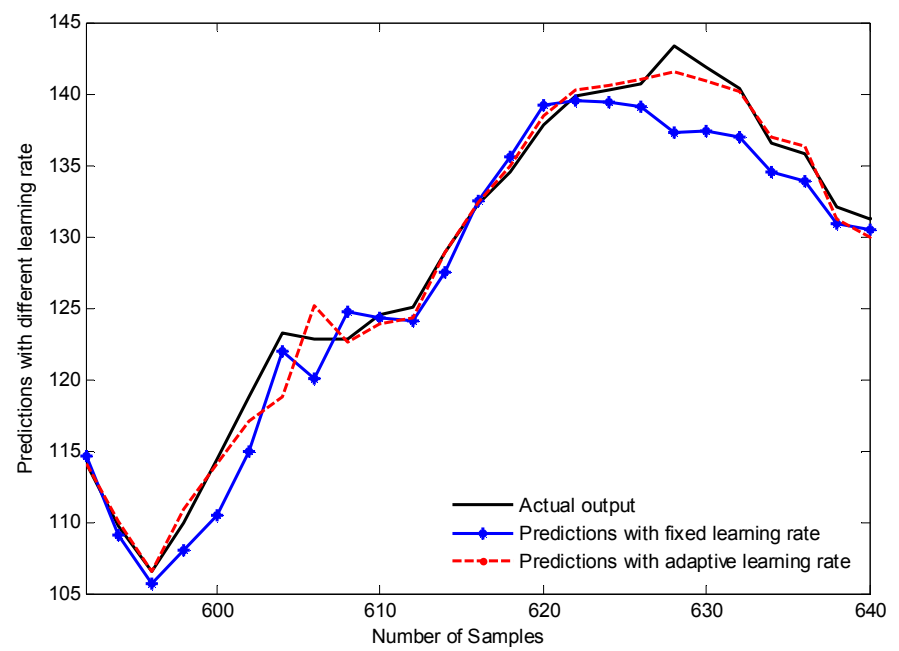

Fig. 11. Predictions of SVI with different learning rates

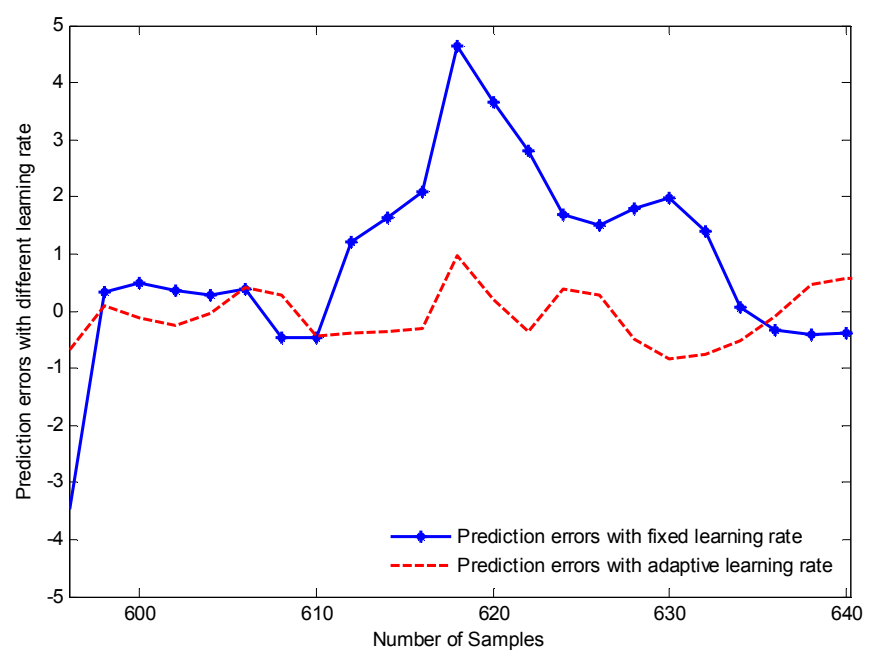

Fig. 12. Predictions errors of SVI with different learning rates

TABLE 4. INPUTS USED IN THIS STUDY

\begin{tabular}{ccc}
\hline Variables & Explanation & Main apparatus and instruments \\
\hline $\mathrm{Q}_{\text {in }}(\mathrm{L} / \mathrm{h})$ & Influent flow rate & Flowmeter WL-1A \\
$\mathrm{BOD}(\mathrm{mg} / \mathrm{L})$ & Biological oxygen demand & BSD/BOD 7400 \\
$\mathrm{COD}(\mathrm{mg} / \mathrm{L})$ & Chemical oxygen demand & COD 5B-3C \\
$\mathrm{DO}(\mathrm{mg} / \mathrm{L})$ & Dissolved oxygen concentration & WTW oxi/340i oxygen probe \\
$\mathrm{pH}$ & Acidity and basicity & WTW inoLab $\mathrm{pH}$ level2 \\
$\mathrm{TN}(\mathrm{mg} / \mathrm{L})$ & Total nutrients & Multi N/C 3000 TOC/TN \\
\hline
\end{tabular}

From Table 5, it is seen that higher mean accuracy is achieved by the proposed ASOA-FNN model with adaptive learning rate (mean accuracy value is $98.65 \%$ ). Meanwhile, Table 5 illustrates that the mean accuracy by the proposed ASOS-FNN model is higher than those in other methods, which also shows that the proposed ASOA-FNN with adaptive learning rate may 
increase the chance to approximate the global optimization parameters in the learning process. Furthermore, the results demonstrate that the SVI trends in the wastewater treatment processes can be predicted with acceptable accuracy by using the $Q_{i n}, \mathrm{BOD}, \mathrm{COD}, \mathrm{DO}, \mathrm{pH}$, and TN data as input variables, which has been proven in [38].

TABLE 5. A COMPARISON OF THE PERFORMANCE OF DIFFERENT METHODS (ALL RESULTS WERE AVERAGED ON 50 INDEPENDENT RUNS)

\begin{tabular}{ccc}
\hline Algorithms & Testing RMSE & Mean Accuracy $(\%)$ \\
\hline ASOS-FNN (Adaptive learning rate $\lambda(\mathbf{0})=\mathbf{0 . 0 1})$ & $\mathbf{0 . 1 5 2}$ & $\mathbf{9 8 . 6 5}$ \\
\hline ASOA-FNN (Fixed learning rate $\lambda=0.01)$ & 0.167 & 98.60 \\
ASOA-FNN (Fixed learning rate $\lambda=0.05)$ & 0.165 & 98.61 \\
ASOA-FNN (Fixed learning rate $\lambda=0.001)$ & 0.172 & 98.58 \\
ASOA-FNN (Fixed learning rate $\lambda=0.0005)$ & 0.166 & 98.61 \\
\hline FNN [7] & $0.286^{*}$ & $94.52^{*}$ \\
ISO-FNN algorithm [32] & 0.248 & 95.53 \\
SORBFNN method [38] & $0.188^{*}$ & $98.10^{*}$ \\
\hline
\end{tabular}

* The results are listed in the original papers.

\section{Conclusion}

In this paper, an ASOA-FNN, based on the second-order algorithm and the adaptive learning rate strategy, has been proposed for training the parameters of FNN. In this ASOA-FNN, the parameters have been updated by the sum of related quasi Hessian matrix and gradient vector as well as with an adaptive learning rate. Moreover, the convergence of ASOA-FNN has been proved. And a proof shows that the adaptive learning rate strategy can accelerate the convergence. In fact, the proposed ASOA-FNN is firstly put forward and tested to model the classical nonlinear dynamic system and predict the sludge bulking in the wastewater treatment processes. The results indicates that the proposed ASOA-FNN owns some advantages for modeling nonlinear systems in terms of good testing RMSE, testing APE, as well as high mean accuracy, etc. The comparisons of the three examples demonstrate that the learning efficiency and performance of ASOA-FNN are better than that of some well-known other algorithms.

In industrial applications, the proposed ASOA-FNN method is better at predicting the sludge bulking in the wastewater treatment processes. The control efficiency of the wastewater treatment system can be further improved by tuning the operating parameters according to the predicted results. In addition, for the real application, a self-organizing strategy will be developed for the proposed ASOA-FNN method. A new self-organizing ASOA-FNN, which can yield more accurate predictions, accelerate the convergence rate, and improve the robustness than the fixed models, will be discussed in the future. 


\section{ACKNOWLEDGMENT}

The authors would like to thank Dr. J. Guo for reading the manuscript and providing valuable comments. The authors also would like to thank the anonymous reviewers for their valuable comments and suggestions, which helped improve this paper greatly.

\section{REFERENCES}

[1] M. M. Ebadzadeh, A. Salimi-Badr, CFNN: Correlated fuzzy neural network, Neurocomputing 148 (1) (2015) $430-444$.

[2] M. Pratama, M. J. Er, X. Li, X. Li, J. Oentaryo, E. Lughofer, I. Arifin, Data driven modeling based on dynamic parsimonious fuzzy neural network, Neurocomputing 110 (1) (2013) 18-28

[3] J.F. Qiao, H.G. Han. Identification and modeling of nonlinear dynamical systems using a novel self-organizing RBF-based approach, Automatica 48 (8) (2012) $1729-1734$.

[4] J.F. Wu, Y.Z. Li, D.E. Quevedo, V. Lau, L. Shi, Data-driven power control for state estimation: a Bayesian inference approach, Automatica 54 (1) (2015) 332-339.

[5] K. Cpałka, K. Lapa, A. Przybył, M. Zalasiński, A new method for designing neuro-fuzzy systems for nonlinear modelling with interpretability aspects, Neurocomputing 135 (1) (2014) 203-217.

[6] D. Bhattacharya, A. Konar, P. Das, Secondary factor induced stock index time-series prediction using Self-Adaptive Interval Type-2 Fuzzy Sets, Neurocomputing 171 (1) (2016) 551-568.

[7] H.G. Han, Y. Li, J.F. Qiao, A fuzzy neural network approach for online fault detection in waste water treatment process, Computers and Electrical Engineering 40 (7) (2014) 2216-2226.

[8] X. Wang, J.Z. Yu, C.D. Li, H. Wang, T.W. Huang, J.J. Huang, Robust stability of stochastic fuzzy delayed neural networks with impulsive time window, Neural Networks 67 (1) (2015) 84-91.

[9] H.G. Han, X.L. Wu, J.F. Qiao, Nonlinear systems modeling based on self-organizing fuzzy-neural-network with adaptive computation algorithm, IEEE Transactions on Cybernetics 44 (4) (2014) 554-564.

[10] D. Meng, Z. Pei, Dynamic adaptive learning algorithm based on two-fuzzy neural-networks, Neurocomputing 125 (1) (2014) 88-94.

[11] P.Y. Liu, H.X. Li, Efficient learning algorithms for three-layer regular feedforward fuzzy neural networks, IEEE Transactions on Neural Networks 15 (3) (2004) 545-558.

[12] S.T. Tzeng, Design of fuzzy wavelet neural networks using the GA approach for function approximation and system identification, Fuzzy Sets and Systems 161 (19) (2010) 2585-2596.

[13] M.R. Mashinchi, A. Selamat, An improvement on genetic-based learning method for fuzzy artificial neural networks, Applied Soft Computing 9 (4) (2009) 1208-1216.

[14] R.J. Kuo, S.Y. Hung, W.C. Cheng, Application of an optimization artificial immune network and particle swarm optimization-based fuzzy neural network to an RFID-based positioning system, Information Sciences 262 (3) (2014) 78-98.

[15] H. Melo, J. Watada, Gaussian-PSO with fuzzy reasoning based on structural learning for training a neural network, Neurocomputing 172 (1) (2016) $405-412$.

[16] C.H. Chen, C.J. Lin, C.T. Lin, Nonlinear system control using adaptive neural fuzzy networks based on a modified differential evolution, IEEE Transactions on Systems, Man, and Cybernetics-Part C: Application and Reviews 39 (4) (2009) 459-473.

[17] R.A. Alieva, B.G. Guirimova, B. Fazlollahib, R.R. Alievc, Evolutionary algorithm-based learning of fuzzy neural networks. Part 2: Recurrent fuzzy neural networks, Fuzzy Sets and Systems 160 (17) (2009) 2553-2566.

[18] S. C. Tan, J. Watada, Z. Ibrahim, M. Khalid, Evolutionary fuzzy ARTMAP neural networks for classification of semiconductor defects, IEEE Transactions on Neural Networks and Learning Systems, 26 (5) (2015) 933-950.

[19] K. Dahal, K. Almejalli, M.A. Hossain, W.B. Chen, GA-based learning for rule identification in fuzzy neural networks, Applied Soft Computing 35 (1) (2015) 605-617.

[20] D. Meng, Z. Pei, Dynamic adaptive learning algorithm based on two-fuzzy neural-networks, Neurocomputing 125 (1) (2014) 88-94.

[21] C.H. Lee, C.T. Li, F.Y. Chang, A species-based improved electromagnetism-like mechanism algorithm for Tsk-Type interval-valued neural fuzzy system optimization, Fuzzy Sets and Systems 171(1) (2011) 22-43.

[22] W. Zhao, K. Li, G.W. Irwin, A new gradient descent approach for local learning of fuzzy neural models, IEEE Transactions on Fuzzy Systems 21 (1) (2013) 30-44.

[23] S. Yilmaz, Y. Oysal, Fuzzy wavelet neural network models for prediction and identification of dynamical systems, IEEE Transactions on Neural Networks 21(10) (2010) 1599-1609.

[24] C.L. Chen, J. Wang, C.H. Wang, L. Chen, A new learning algorithm for a fully connected neuro-fuzzy inference system, IEEE Transactions on Neural Networks and Learning Systems 25 (10) (2014) 1741-1757.

[25] M.M. Ebadzadeh, A. Salimi-Badr, CFNN: Correlated fuzzy neural network, Neurocomputing 148 (1) (2015) $430-444$.

[26] J. A. Sanz, D. Bernardo, F. Herrera, H. Bustince, H. Hagras, A compact evolutionary interval-valued fuzzy rule-based classification system for the modeling and prediction of real-world financial applications with imbalanced data, IEEE Transactions on Fuzzy Systems, 23 (4) (2015) 973-990.

[27] H.G. Han, J.F. Qiao, A self-organizing fuzzy neural network based on a growing-and-pruning algorithm, IEEE Transactions on Fuzzy Systems 18 (6) (2010) 1129-1143.

[28] J.Y. Fan, J.L. Zeng, A Levenberg-Marquardt algorithm with correction for singular system of nonlinear equations, Applied Mathematics and Computation 219 (17) (2013) 9438-9446.

[29] B.M. Wilamowski, H. Yu, Improved computation for Levenberg-Marquardt training, IEEE Transactions on Neural Networks 21 (6) (2010) 930-937.

[30] N. Ampazis, S.J. Perantonis, Two highly efficient second-order algorithms for training feedforward networks, IEEE Transactions on Neural Networks 13 (5) (2002) 1064-1074.

[31] C.H. Lu, Wavelet fuzzy neural networks for identification and predictive control of dynamic systems, IEEE Transactions on Industrial Electronics 58 (7) (2011) 3046-3058.

[32] B. Pizzileo, K. Li, G.W. Irwin, W.Q. Zhao, Improved structure optimization for fuzzy-neural networks, IEEE Transactions on Fuzzy Systems 20 (6) (2012) 1076-1089. 
[33] M. Davanipoor, M. Zekri, F. Sheikholeslam, Fuzzy wavelet neural network with an accelerated hybrid learning algorithm, IEEE Transactions on Fuzzy Systems 20 (3) (2012) 463-470.

[34] N. Wang, J.E. Meng, X.Y. Meng, A fast and accurate online self-organizing scheme for parsimonious fuzzy neural networks, Neurocomputing 72 (16-18) (2009) 3818-3829.

[35] C.F. Juang, C.D. Hsieh, TS-fuzzy system-based support vector regression, Fuzzy Sets and Systems 160 (17) (2009) $2486-2504$.

[36] N. Eshtiaghi, F. Markis, S.D. Yap, J.C. Baudez, P. Slatter, Rheological characterization of municipal sludge: A review, Water Research 47 (15) (2013) 5493-5510.

[37] C.G. Martínez-Garcia, M.T. Olguín, C. Fall, Aerobic stabilization of biological sludge characterized by an extremely low decay rate: modeling, identifiability analysis and parameter estimation, Bioresource Technology 166 (8) (2014) 112-119.

[38] H.G. Han, J.F. Qiao, Prediction of activated sludge bulking based on a self-organizing RBF neural network, Journal of Process Control 22 (6) (2012) 1103-1112.

This work was supported by the National Science Foundation of China under Grants 61533002 and 61225016 , Beijing Nova Program under Grant Z131104000413007, China Postdoctoral Science Foundation under Grant 2014M550017, Ph.D. Program Foundation from Ministry of Chinese Education under Grant 20121103120020 and 20131103110016, Collaborative Innovation Program under Grant ZH14000177, Project supported by Beijing Postdoctoral Research Foundation under Grant 2015ZZ-03, and Beijing Municipal Education Commission Foundation under Grants km201410005001 and KZ201410005002. Asterisk indicates corresponding author. 
Honggui Han received the B.E. degree in automatic from Civil Aviation University of China, Tianjin, China, in 2005, the M.E. and Ph.D. degrees from the Beijing University of Technology, Beijing, China, in 2007 and 2011, respectively.

He has been with Beijing University of Technology since 2011, where he is currently a Professor. His current research interests include neural networks, fuzzy systems, intelligent systems, modeling and control in process systems, and civil and environmental engineering.

Prof. Han is a member of the IEEE Computational Intelligence Society. He is currently a reviewer of IEEE Transactions on Fuzzy Systems, IEEE Transactions on Neural Networks and Learning Systems, IEEE Transactions on Cybernetics, IEEE Transactions on Control Systems Technology, Control Engineering Practice, and Journal of Process Control.

Luming Ge received B.E. degree in automatic control from University of Science and Technology Liaoning, Anshan, China, in 2013. He is currently pursuing the M.E. degree with the College of Electronic and Control Engineering, Beijing University of Technology, Beijing, China.

His current research interests include neural networks, intelligent systems, modeling and control in process systems.

Junfei Qiao received B.E. and M.E. degrees in control engineer from Liaoning Technical University, Fu'xin, China, in 1992 and 1995, respectively; and the Ph.D. degree from Northeast University, Shenyang, China, in 1998.

From 1998 to 2000, he was a Postdoctoral Fellow with the School of Automatics, Tianjin University, Tianjin, China. He joined Beijing University of Technology, where he is currently a Professor. He is the director of the intelligence systems lab. His research interests include neural networks, intelligent systems, self-adaptive/learning systems, and process control systems.

Prof. Qiao is a member of the IEEE Computational Intelligence Society. He is currently a reviewer for more than 20 international journals, such as IEEE Transactions on Fuzzy Systems, IEEE Transactions on Neural Networks and Learning Systems. 
${ }^{*}$ Photo of the author(s)
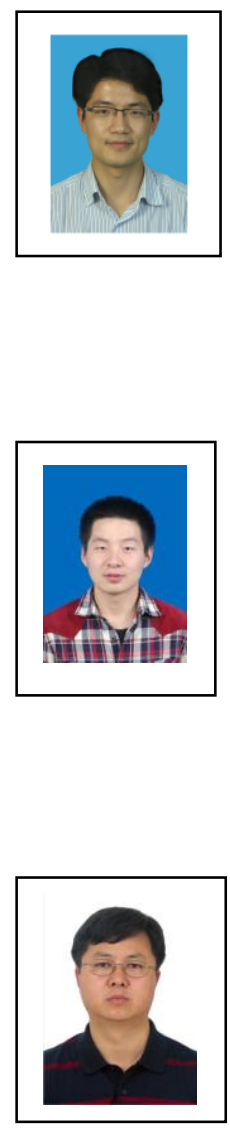